\title{
Large Retroperitoneal Hematoma and Lumbosacral Plexopathy Following Microsurgical Decompression via the Wiltse Paraspinal Approach: A Case Report
}

\author{
Tsz-King Suen ${ }^{1}$, Guang-Xun Lin ${ }^{1}$, Hyun-Jin $\mathrm{Jo}^{1}$, Javier Quillo-Olvera ${ }^{1}$, Jin-Sung Kim ${ }^{1,2}$ \\ ${ }^{1}$ Department of Neurosurgery \& ${ }^{2}$ The Catholic Central Laboratory of Surgery (CCLS), Seoul St. Mary's Hospital, College of Medicine, \\ The Catholic University of Korea, Seoul, Korea
}

Corresponding Author: Jin-Sung Kim, $\mathrm{MD}, \mathrm{PhD}$

Department of Neurosurgery, Seoul

St. Mary's Hospital, College of Medicine,

The Catholic University of Korea, 222

Banpo-daero, Seocho-gu, Seoul 06591, Korea

Tel: $+82-2-2258-6128$

Fax: +82-2-2258-6128

E-mail: mdlukekim@gmail.com

Received: February 26, 2018

Revised: October 11, 2018

Accepted: October 12, 2018

\begin{abstract}
Paraspinal approaches are associated with fewer complications and better outcomes than midline approaches. However, most surgeons are not conversant with the surgical anatomy in these approaches, especially at the lumbosacral junction, where the anatomy is more complex. This paper reports a case of concomitant vascular and neurological complication following the use of the microscopic Wiltse approach. A 71-year-old woman presented with symptoms of spinal stenosis and right L5 radiculopathy. She underwent microsurgical decompression of a foraminal stenosis at the lumbosacral junction via the Wiltse approach, and a midline decompression from L3 to L5. The tubular retractor was docked inappropriately, being too deep and lateral. It directly punctured through the intertransverse membrane and injured the retroperitoneal segmental vessel and lumbosacral plexus. Postoperatively, she developed a large retroperitoneal hematoma and lumbosacral plexopathy, which were treated with surgery and intensive rehabilitation. This report highlights the importance of accurate retractor docking, familiarity with the surgical anatomy, and recognition of the potential complications of this technique.
\end{abstract}

Key Words: Decompression, Hematoma, Paraspinal

\section{INTRODUCTION}

Foraminal or extraforaminal disc herniation and stenosis are being encountered more frequently because of advances in imaging technology. Nerve root compression usually occurs at the dorsal root ganglion, causing intractable pain and neurologic deficits. Various paraspinal approaches are used for treating this condition ${ }^{11,17)}$, and are associated with fewer complications and better outcomes than midline approaches with respect to decompression, preservation of facets, and spinal stability ${ }^{14}$. However, most surgeons are not conversant with the surgical anatomy of these approaches, especially at the lumbosacral junction, where the anatomy is more complex. This can result in inadequate decompression or vascular injuries. To our knowledge, this is the first report of a case of concomitant vascular and neurological complication following the use of the microscopic Wiltse approach. Informed consent was obtained from the patient for the publication of this report, including the necessary photographs.

\section{CASE REPORT}

A 71-year-old woman presented with a 3-month history of right lateral leg pain without significant back pain. She subsequently developed symptoms of spinal claudication at a walking distance of 100 meters one month after the initial presentation. Conservative management with physiotherapy and analgesics was unsuccessful. Physical examination revealed grade 4 weakness in the right ankle and right great toe in dorsiflexion, and a sensory deficit at the right L5 dermatomal distribution. Magnetic resonance imaging (MRI) demonstrated central spinal stenosis from L3 to S1 and right L5-S1 foraminal stenosis (Fig. 1).

Radiography revealed mild degenerative lumbar scoliosis with no definite instability. Preoperative electromyography (EMG) suggested a right L5 and S1 radiculopathy, which was further confirmed by positive provocation and symptom relief following a selective right $L 5$ nerve root block with lidocaine and steroids. The surgical plan included an L3-4 and L4-5 microscopic bilateral laminotomy with a tubular retractor via the left midline approach, and an L5-S1 microscopic tubular foraminoplasty via the rightside Wiltse approach.

L3-4 and L4-5 laminotomy was performed uneventfully (Fig. 2). We proceeded on to the right side using the Wiltse paraspinal approach, up to the L5-S1 level. Upon docking the tubular retractor, significant bleeding occurred. The tubular retractor was docked too far laterally and cranially (at the L4-5 level ins- 

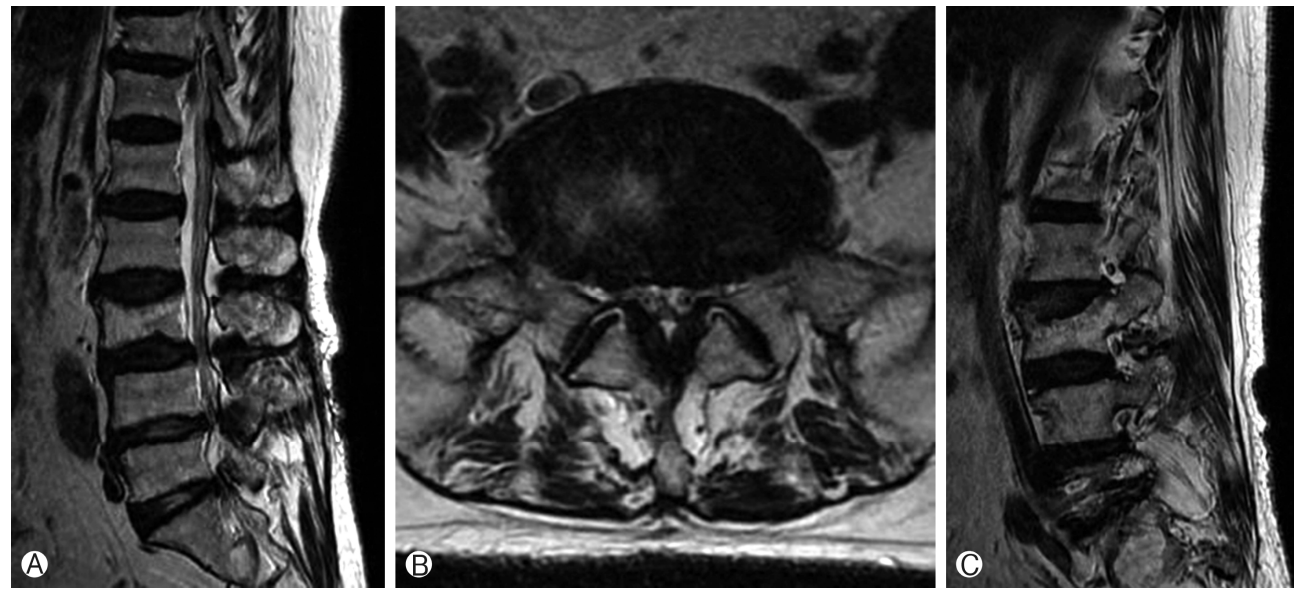

Fig. 1. Preoperative MRI demonstrating L3 to S1 central spinal stenosis and right L5-S1 foraminal stenosis. (A) Mid-sagittal view; (B) L5-S1 transverse cut; (C) Right foraminal region.

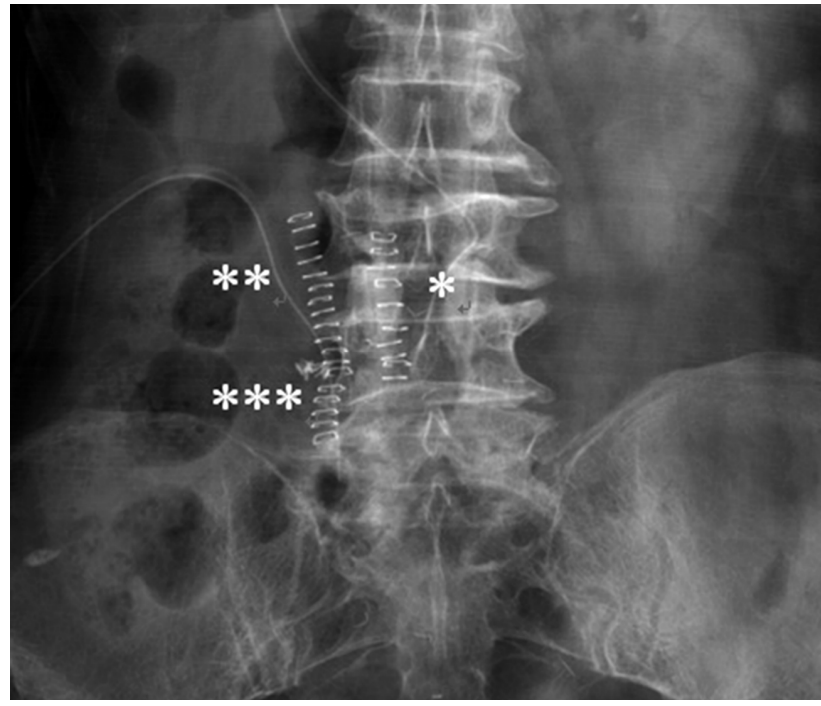

Fig. 2. Postoperative radiograph showing the incision of $L 3-4$ and L4-5 laminotomy $\left({ }^{*}\right)$, the initial docking for the lumbosacral paraspinal decompression $\left(^{* *}\right)$ being too lateral and cranial, and the final docking for the lumbosacral decompression $\left.{ }^{* *}\right)$.

tead of towards the lumbosacral junction). The bleeding originated from the right $L 4$ segmental artery. This artery was coagulated and ligated with a hemoclip, and the tubular retractor was repositioned. Subsequent foraminal decompression of the right L5-S1 level was performed and the right L5 nerve root was released.

Postoperatively, the patient was found to have diffuse, marked, grade 1 weakness from the right L2 to L5 myotome. Immediate postoperative MRI (Fig. 3) showed a huge hematoma $(8 \times 5 \times 5 \mathrm{~cm})$ extending from the L3 to L5 level within and adjacent to the psoas muscle and lumbosacral plexus. Postoperative computed tomography (CT) (Fig. 4) showed adequate bony decompression in both the central canal, from $L 3$ to $L 5$, and the right L5-S1 foramen, with a surgical hemoclip at the L4-5 level. She
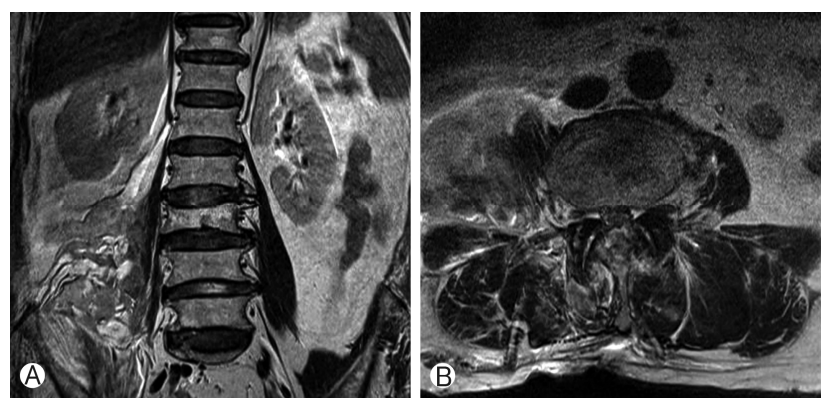

Fig. 3. Postoperative MRI showing huge hematoma formation within and adjacent to the psoas muscle. (A) Coronal cut; (B) Transverse cut.
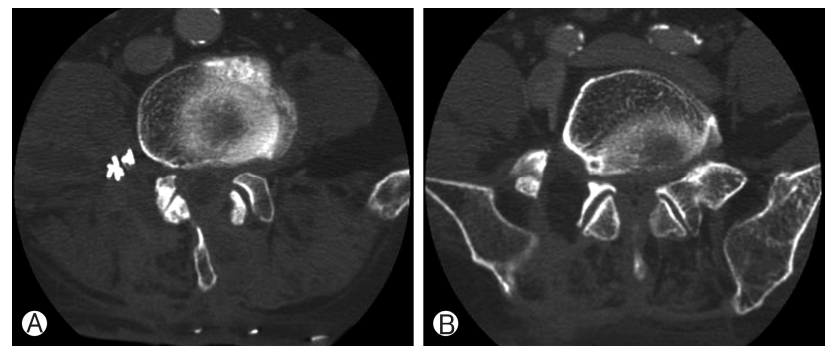

Fig. 4. Postoperative CT scan showing adequate bony decompression of the central canal from L3 to L5 and the right L5-S1 foramen and surgical hemoclip at the L4-5 level. (A) L4-5 level; (B) Lumbosacral junction.

underwent surgical removal of the huge hematoma in the retroperitoneal space the following day, with no active bleeding identified. Although the hematoma appeared largely resolved $(4.5 \times 2 \times 2 \mathrm{~cm})$ on a contrast CT image taken 6 weeks after her initial surgery, EMG performed 1 and 3 months postoperatively showed right diffuse lumbosacral polyradiculopathy with severe axonopathy with little interval change. She reported neuropathic pain over her right $L 2$ and $L 3$ dermatomes, requiring buprenor- 
phine treatment. She was also diagnosed with depression and was treated with venlafaxine. After one year of inpatient intensive rehabilitation, her motor power gradually improved to grade 3 and she was able to walk with a cane for 200 meters.

\section{DISCUSSION}

Three paraspinal approaches to the lumbar spine have been described in the literature. The approaches described by Ray and Watkins were between the sacrospinalis muscle and the quadratus lumborum muscle ${ }^{11,17)}$. The more medial sacrospinalis muscle-splitting approach (between the multifidus and longissimus) was initially described and popularized by Wiltse ${ }^{177}$. This technique was initially developed for lumbar arthrodesis. The indications for this approach were subsequently extended to include treatments of far lateral disc herniation, far out syndrome, foraminal stenosis, pedicle screw insertion, and transforaminal lumbar interbody fusion ${ }^{3,5,18)}$. The advantage of this approach stems from its muscle-splitting technique, which prevents muscle damage. In degenerative conditions of the spine, this technique demonstrated a decreased risk of wound breakdown, postoperative infection, blood loss, and reoperation ${ }^{14)}$. Subsequent modification of this approach led to an even less-invasive technique using microscopy and tubular microendoscopy $^{4}$. Despite the trend towards minimally invasive procedures, a thorough understanding of surgical anatomy is important to avoid complications. The present case demonstrates that the minimally invasive paraspinal approach can still result in major vascular and neurological complications.

The natural cleavage plane between the multifidus and longissimus is more easily identified at a more caudal level, but vascularity increases in the caudal direction ${ }^{15)}$. The medial-lateral skin entry point could be identified by measuring the distance from the midline of the cleavage plane on $\mathrm{MR}^{16}{ }^{16}$. In the open technique, the cleavage plane is guided by the identification of the perforating vessels ${ }^{15}$. In our case, at the level of the lumbosacral junction, the anatomy posed additional surgical challenges. At the lumbosacral junction, there are rigid bony confines due to a wider pars interarticularis at $L 5$, a shorter distance from the $L 5$ transverse process to the superior edge of the superior articulating process, and a coronally oriented L5-S1 facet and iliac crest ${ }^{12)}$.

One paper described an open approach to the lumbosacral junction by initiating the dissection of the lumbodorsal fascia from the iliac crest, and the identification of the L5 transverse process, the iliolumbar ligament, and the lateral edge of the L5-S1 facet ${ }^{7}$. Another study described a serial dilation technique, in which the author suggested making a 3-cm skin incision $1-\mathrm{cm}$ lateral to the medial border of the $L 5$ transverse process, allowing an angled trajectory with subsequent dissection inferiorly to the pars, facet, and sacral ala ${ }^{9}$. CT-navigated tubular decompression could also aid in optimizing the trajectory, preserving the facet, and minimizing drilling in cases of foraminal stenosis ${ }^{13)}$.

In our case, the entry point was wrongly judged at the L4-5
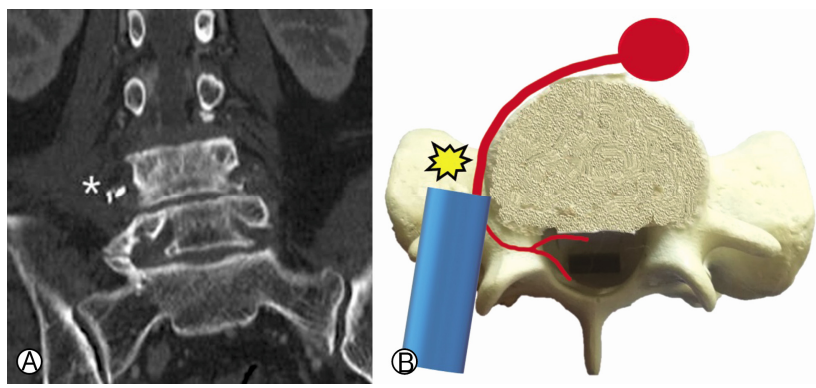

Fig. 5. Inappropriate docking of the tubular retractor could lead to direct puncture through the intertransverse membrane and injure the retroperitoneal segmental vessel or its branches. (A) The position of the hemoclip $\left(^{*}\right)$; (B) The retractor abutting the segmental vessel and its branch

level. The cephalic entry point of the dilator may be caused by blockage from the iliac crest. Without properly docking the retractor in the above-mentioned bony structures ( $L 5$ transverse process and L5-S1 facet) as a reference point, the tubular retractor could directly puncture through the intertransverse membrane and injure the retroperitoneal segmental vessels and lumbosacral plexus (Fig. 5). This resulted in a large retroperitoneal hematoma and lumbosacral plexopathy in this patient.

After docking the retractor, further exposure is required by drilling off the lower border of the $L 5$ transverse process and the superolateral part of the S1 superior articular process, followed by resection of the lateral part of the isthmus and the superomedial part of the S1 superior articular process ${ }^{2)}$. The intertransverse and foraminal ligaments are then released to decompress the $L 5$ exiting nerve.

Vascular injuries (acute or delayed) can occur in all types of spinal approaches. These are particularly well-studied using the anterior direct approach, due to its proximity to major vessels (namely, the iliac vessels and inferior vena cava), with the associated risk of mortality. The overall risk of vascular injuries was reported to be 0 to $18 \% \%^{6}$. The risk of vascular injuries in oblique interbody fusion was reported to be lower, at 2.6\% for the segmental artery and $1.3 \%$ for other arteries ${ }^{1)}$. For direct posterior discectomy, the rate was reported to be 0.01 to $0.05 \%{ }^{10)}$. However, as injuries were usually of major vessels (aorta, iliac vessels, inferior vena cava) in front of the anterior annulus, the presentation could be delayed and result in mortality (up to $10 \%)^{10}$. For direct lateral and paramedian approaches, only isolated cases of vascular injury have been reported. These injuries were usually at the segmental arteries or veins, with symptoms of flank and leg edema.

To our knowledge, this is the first report of combined complications of retroperitoneal hematoma with significant lumbosacral plexopathy. The anatomy of the posterior branches of the segmental vessels has been well-described in the literature ${ }^{8}$. The segmental vessels emerge from the aorta towards to the inferior edge of the pedicles, lateral to the exiting nerve roots. They divide into five posterior branches: (1) to the anterior surface of the transverse process, (2) around the waist of the pars, 
(3) between the multifidus and longissimus, (4) into the midlateral aspect of the erector spinae, and (5) travelling with the lateral branch of the posterior primary ramus.

The presented complications could be avoided by (1) correctly locating the docking point by preoperative planning using MRI and fluoroscopic guidance for the insertion of the guidewire, (2) gentle finger dissection to the transverse process and facet joint, and (3) drilling of the bone and releasing the intertransverse and foraminal ligaments under direct microscopic vision. In the case of extraforaminal stenosis, further caution is required because of the more ventral location of the pathology and higher risk of vascular injury.

\section{CONCLUSION}

The paraspinal approach is an attractive surgical approach, especially when combined with minimally invasive techniques. However, good knowledge of surgical anatomy, preoperative planning, and gentle operative techniques with good illumination under magnification are essential for achieving good decompression and preventing surgical complications.

\section{REFERENCES}

1. Abe $\mathrm{K}$, Orita S, Mannoji $\mathrm{C}$, Motegi $\mathrm{H}$, Aramomi M, Ishikawa $\mathrm{T}$, et al: Perioperative complications in 155 patients who underwent oblique lateral interbody fusion surgery: perspectives and indications from a retrospective, multicenter survey. Spine (Phila Pa 1976) 42:55-62, 2017

2. Cho SI, Chough CK, Choi SC, Chun JY: Microsurgical foraminotomy via Wiltse paraspinal approach for foraminal or extraforaminal stenosis at L5-S1 level: risk factor analysis for poor outcome. J Korean Neurosurg Soc 59:610-614, 2016

3. Fankhauser H, de Tribolet N: Extreme lateral lumbar disc herniation. Br J Neurosurg 1:111-129, 1987

4. Foley KT, Smith MM, Rampersaud YR: Microendoscopic approach to far-lateral lumbar disc herniation. Neurosurg Focus 7:e5, 1999

5. Guyer DW, Wiltse LL, Peek RD: The Wiltse pedicle screw fixation system. Orthopedics 11:1455-1460, 1988

6. Inamasu J, Guiot BH: Vascular injury and complication in neurosurgical spine surgery. Acta Neurochir (Wien) 148:375-387,
2006

7. Kotil K, Akcetin M, Bilge T: A minimally invasive transmuscular approach to far-lateral L5-S1 level disc herniations: a prospective study. J Spinal Disord Tech 20:132-138, 2007

8. O'Brien MF, Peterson D, Crockard HA: A posterolateral microsurgical approach to extreme-lateral lumbar disc herniation. J Neurosurg 83:636-640, 1995

9. O'Toole JE, Eichholz KM, Fessler RG: Minimally invasive far lateral microendoscopic discectomy for extraforaminal disc herniation at the lumbosacral junction: cadaveric dissection and technical case report. Spine J 7:414-421, 2007

10. Papadoulas S, Konstantinou D, Kourea HP, Kritikos N, Haftouras N, Tsolakis JA: Vascular injury complicating lumbar disc surgery. a systematic review. Eur J Vasc Endovasc Surg 24: 189195, 2002

11. Ray CD: The paralateral approach to decompression for lateral stenosis and far lateral lesions of the lumbar spine. In: Watkins E, ed. Principles and Techniques in Spine Surgery. Aspen, CO: Collis, 217-227, 1987

12. Reulen HJ, Muller A, Ebeling U: Microsurgical anatomy of the lateral approach to extraforaminal lumbar disc herniations. Neurosurgery 39:345-350; discussion 350-341, 1996

13. Stavrinou P, Hartl R, Krischek B, Kabbasch C, Mpotsaris A, Goldbrunner R: Navigated transtubular extraforaminal decompression of the L5 nerve root at the lumbosacral junction: clinical data, radiographic features, and outcome analysis. Biomed Res Int 2016:3487437, 2016

14. Street JT, Andrew Glennie R, Dea N, DiPaola C, Wang Z, Boyd $\mathrm{M}$, et al: A comparison of the Wiltse versus midline approaches in degenerative conditions of the lumbar spine. J Neurosurg Spine 25:332-338, 2016

15. Vialle R, Court C, Khouri N, Olivier E, Miladi L, Tassin JL, et al: Anatomical study of the paraspinal approach to the lumbar spine. Eur Spine J 14:366-371, 2005

16. Warren A, Prasad V, Thomas M: Pre-operative planning when using the Wiltse approach to the lumbar spine. Ann R Coll Surg Engl 92:74-75, 2010

17. Watkins MB: Posterolateral bonegrafting for fusion of the lumbar and lumbosacral spine. The Journal of Bone and Joint Surgery American 41-A:388-396, 1959

18. Wiltse LL, Guyer RD, Spencer CW, Glenn WV, Porter IS: Alar transverse process impingement of the L5 spinal nerve: the far-out syndrome. Spine (Phila Pa 1976) 9:31-41, 1984 